\title{
A Conceptual Investigation of a Large Radio Telescope Support Point Number Effect on Its Pointing Accuracy
}

\author{
Song Xue $\mathbb{D}^{\mathrm{D}},{ }^{1}$ Kaitong Ma, ${ }^{1}$ Congsi Wang $\mathbb{D}^{1},{ }^{1}$ Peiyuan Lian, ${ }^{1}$ Yan Wang, ${ }^{2}$ Yuefei Yan, \\ Qian Xu, ${ }^{3}$ Yuanpeng Zheng, ${ }^{4}$ and Na Wang ${ }^{3}$ \\ ${ }^{1}$ Key Laboratory of Electronic Equipment Structure Design, School of Mechano-Electronic Engineering, Xidian University, Xi'an, \\ Shaanxi, China \\ ${ }^{2}$ School of Information and Control Engineering, University of Architecture and Technology, Xi'an, Shaanxi, China \\ ${ }^{3}$ Xinjiang Astronomical Observatory, Chinese Academy of Sciences, Urumqi 830011, China \\ ${ }^{4}$ CETC No. 54 Research Institute, Shijiazhuang 050081, China
}

Correspondence should be addressed to Congsi Wang; congsiwang@163.com

Received 30 July 2020; Revised 6 October 2020; Accepted 4 November 2020; Published 23 November 2020

Academic Editor: Francesco D’Agostino

Copyright (C) 2020 Song Xue et al. This is an open access article distributed under the Creative Commons Attribution License, which permits unrestricted use, distribution, and reproduction in any medium, provided the original work is properly cited.

\begin{abstract}
The purpose of this paper is to investigate a large radio telescope support point number effect on its pointing accuracy and provide a useful guideline for the large radio telescope design engineer. In a large radio telescope system, the azimuth track is used to support the whole telescope structure and the mounting error as well as the telescope wheel-track contact in a long term can cause unevenness on the azimuth track, which can further deteriorate the telescope pointing accuracy. Even though various compensation methods have been proposed to compensate for this pointing error, it remains as one of the challenges for the telescope pointing error reduction. In this paper, a general telescope pointing error estimation formula has been proposed to investigate different telescope support-point number designs on its pointing accuracy. In this approach, the azimuth track unevenness has been modelled as the Fourier function using the least square method after the raw track profile has been measured. Next, the elevation position matrix, azimuth position matrix, and the azimuth profile matrix can be constructed for different telescope support point numbers, and the telescope pointing error can then be obtained based on the proposed general formula. The telescope pointing error root mean square (RMS) value is used to quantify the effect of the telescope support point number on the pointing accuracy. Two interesting results can be observed in the numerical example. The first one is that the telescope pointing error curves have different dominant peaks during one azimuth track rotation, which is corresponding to the support point number. Another interesting finding is that the RMS value experienced a complex trend with the support point number change, and they are not a simple monotonous increasing or decreasing relationship with the support number. All the results in this paper can provide a useful guideline for reducing the telescope pointing error in the initial design stage.
\end{abstract}

\section{Introduction}

In 2014, China has proposed to build the Qi Tai Telescope (QTT), which will be a fully steerable telescope with a $110 \mathrm{~m}$ aperture. The whole telescope structure will be around $6000 \mathrm{t}$ in weight and $120 \mathrm{~m}$ in height. When built, this telescope will be the largest fully steerable telescope in the world. In addition, this telescope is also designed to work from $150 \mathrm{MHz}$ to $115 \mathrm{GHz}$, which requires a repeatable pointing accuracy of 1.19 arc sec. Even though Green Bank Telescope and
Effelsberg Telescope have provided lots of useful experience for QTT design engineer [1], improving the pointing accuracy still poses a big challenge and remains one of the most difficult tasks due to the increased telescope structure size and its high pointing accuracy requirement.

There were many factors that can affect the telescope pointing accuracy from different aspects [2], including the antenna control error, the disturbances from the environment, and the antenna structure irregularities. Various compensation methods have been proposed to minimize 
these telescope pointing errors, and an impressive improvement can be observed after the compensation [3-10]. Among all these telescope pointing error sources, the antenna structure irregularities are considered to be the most influential one as they are directly related to the antenna structure itself [2], and if the mechanical system is accurately done, matching the specifications will be easier; otherwise, it may be even impossible. Once being designed and manufactured, it is almost impossible to change the design. In a telescope structure, the telescope azimuth track is used to undertake the whole gross weight of the telescope, which can weigh up to thousands of tons. Currently, the dominant design of the azimuth track is using the completely welded rails, which can reduce the deformations at the rail joint [11]. Despite the high precision and long service life of this type of azimuth rail, the rail unevenness is still inevitable, which can be caused by manufacturing and assembly error, and so on. During the telescope tracking, this unevenness can cause the whole structure tilts and flexible deformation, which can further deteriorate the telescope pointing accuracy [12].

The study on the telescope azimuth track can be classified into the track profile measurement [13-15], the track profile modelling [16], the telescope pointing error considering the track profile, and its corresponding compensation methods. For the measurement, Li used the digital level to detect the azimuth track unevenness for the $25 \mathrm{~m}$ telescope in Xinjiang Observatory [13], and Wang et al. used the Leica NI007 high precision level to measure the track profile for a $50 \mathrm{~m}$ telescope in Beijing [14]. A similar method has been used by Smith to measure the azimuth track profile for Large Millimeter Telescope (LMT) [15]. An alternative method has been proposed by Gawronski, who installed the inclinometers on the telescope structure and established a relation between the inclinometer data the track unevenness [12]. After the measurement, people are also trying to describe the track unevenness data as precisely as possible, and various models have been proposed. Actually, all these unevenness models have been adopted from the models used for the machining as well as the railway applications. In particular, Li proposed a coarse-fine mixed model to describe the rail unevenness, where the Fourier series was used to describe the large-scale deformation and the Weierstrass-Mandelbrot fractal function was used to describe the small-scale deformation [16]. In their research, azimuth track unevenness is found to be an important source that can affect the telescope's pointing accuracy. In 2000, Gawronski et al. derived the algorithms for the telescope pointing error estimation using the inclinometer data because the azimuth track unevenness is a type of repeatable disturbances for the telescope. Later, he also developed a look-up table method to minimize the pointing error [17]. Based on the model developed in [18], Bartosz introduced a new generalized pointing model accounting for the rail irregularities to improve the $32 \mathrm{~m}$ Torun radio telescope. In [19], Kong proposed a telescope pointing model for the telescope with a design of four support points with the consideration of the azimuth track profile. On the other hand, people are also putting continuous effort to improve the azimuth track manufacturing process $[20,21]$. In the early days, most telescope track designs come from the train railway design with an I shape structure [2]. With the increasing pointing accuracy requirement, two representative telescope track designs have been proposed with new welding technologies. The first one is using the full section exothermic welding in Effelsberg Telescope [22]. The second one is using the U-groove partial welding technology, and the typical representative includes the LMT as well as the Tianma Telescope [22].

However, little has been done with regard to the telescope support points, which is in direct contact with the azimuth track. Even though some work has been done to improve the contact between the wheel and track [23], the little result has been reported on how to design the support points in order to reduce the telescope pointing error. One of the fundamental questions that remain unsolved is the number selection of the telescope support points, and there is still no design guideline on how to select the number of support points in terms of reducing the pointing error. This paper aims to fill this gap and investigate the number of telescope support points on the pointing accuracy. In Section 2 , the telescope pointing error mathematical models for different support point numbers have been developed, and then the corresponding numerical process on evaluating the pointing accuracy and the results are presented in Section 3. In Section 4, the numerical results have been summarized and discussed.

\section{Telescope Pointing Error Mathematical Modelling}

2.1. Telescope Track Model. A steerable reflector antenna is designed to concentrate the radiation from the desired direction as effectively as possible in the focal point from both the azimuth and elevation directions. A typical steerable parabolic reflector normally includes a focal point, the supporting truss, the parabolic reflector, receiver, rotating track, counterweight, supporting structure, and the azimuth track, as shown in Figure 1(a). Among all these components, the azimuth track is used to undertake the whole gross weight of the telescope, which can weigh up to a thousand tons. Therefore, the surface of the azimuth track normally experiences serious contact and settling problem, which could lead to the unevenness of the telescope tracking. When the telescope wheels are in contact with an uneven track, the telescope pointing axis would deviate from its original designed position and correspondingly cause the telescope pointing error. As shown in Figure 1(b), the azimuth track normally consists of a wear plate, a base plate, a thick splice plate below the joints between each base plate, and the cementitious grout. A high tensile anchor bolt is usually used to fasten the base plate to the concrete foundation.

2.2. Azimuth Track Unevenness Modelling. In practice, the telescope azimuth track unevenness is mainly induced by two aspects: the deformation and surface wear during the 


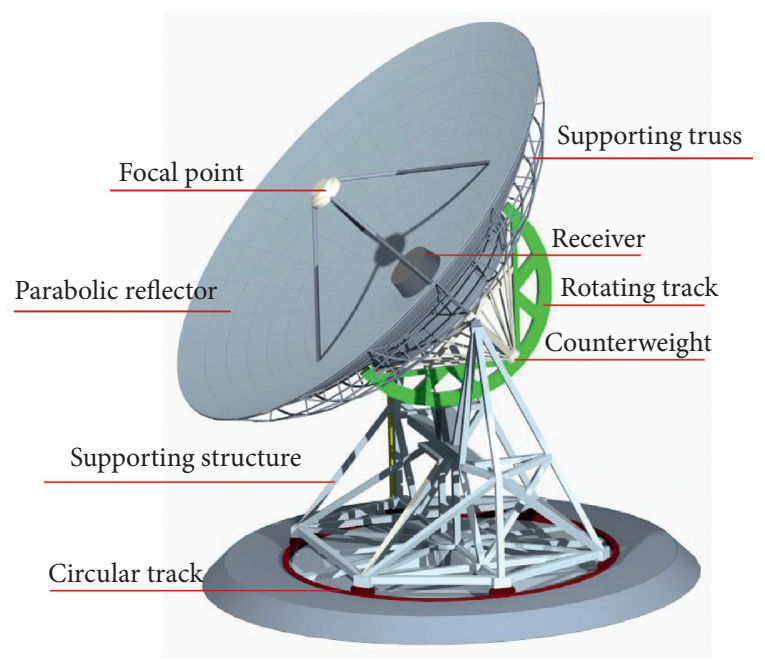

(a)

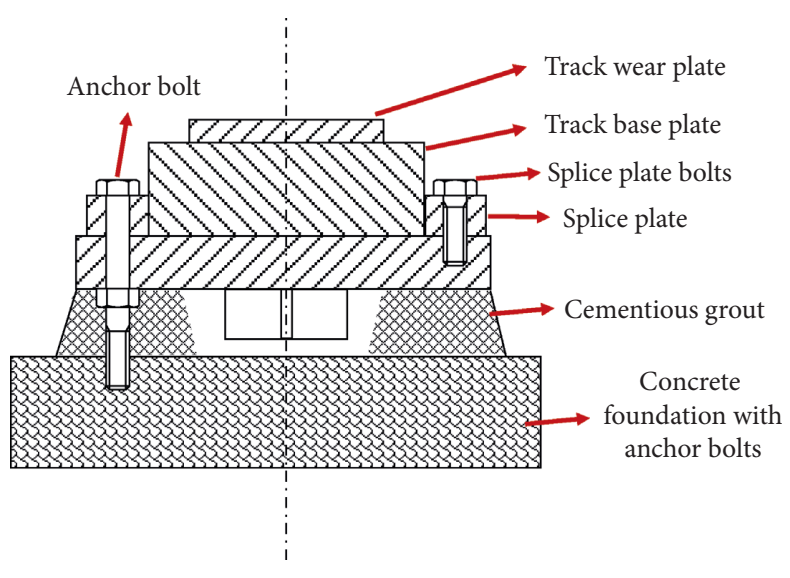

(b)

Figure 1: The typical telescope structure. (a) Steerable parabolic reflector. (b) Azimuth track model.

operation as well as the surface roughness during the machining process [16]. For the first aspect, the unevenness caused by the deformation and surface wear often shows the characteristics with low frequencies and large amplitudes, and this is mainly because the preheating process and temperature control are used during the azimuth track construction process to prevent the appearance of delayed cracks as well as to eliminate quenched structures in the material's heat affected zone after welding, which can lead to rail deformation due to uneven stress distribution. Besides, the whole telescope structure can weigh up to thousands of tons, and the wear behavior between the wheel and the track further deteriorates the track unevenness phenomenon. On the other hand, the unevenness caused by the surface roughness often shows the characteristics with high frequency and low amplitude. This type of unevenness is normally on a smaller scale compared with the first type. An example of the azimuth track unevenness can be found in Figure 2, where a $34 \mathrm{~m}$ telescope azimuth track profile is presented.

Numerous telescope technical reports have shown that the unevenness caused by the track deformation and surface wear has dominated the telescope track profile as they show to be on a much larger scale $[16,19,24]$. Therefore, only the unevenness in these large scales was modelled in this research while the unevenness in the small scale has been neglected even though the inclusion of these small scales could yield a more accurate result [16]. This compromise, on the other hand, can improve the computational speed and the modelling accuracy can still be guaranteed to some extent. In this research, the track profile is a periodic function, and therefore, the Fourier series can be used to represent the track profile error:

$$
f_{1}(x)=a_{0}+\sum_{n=1}^{m}\left[a_{n} \cos \left(n \omega_{0} x\right)+b_{n} \sin \left(n \omega_{0} x\right)\right]
$$

where $x$ is the track circumferential position, $f(x)$ is the azimuth track unevenness value on $x$ position, $a_{0}, a_{n}$, and $b_{n}$

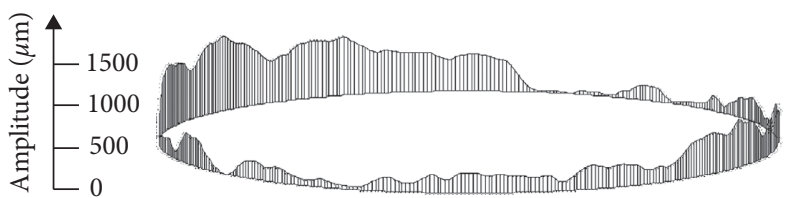

FIgURE 2: The example of the unevenness profile in a $34 \mathrm{~m}$ telescope azimuth track [24].

are the Fourier function coefficients, and $\omega_{0}$ denotes the fundamental frequency of the Fourier function.

2.3. Telescope Coordinate System. In order to quantify the effect of azimuth track on the telescope pointing accuracy, four global and local coordinates established in [16] have been adopted here, and all the detailed information can be found in Figure 3:

(1) OXYZ: the Global coordinate, whose coordinate origin is located on the center of the azimuth track, $Z$ axis is perpendicular to the ground, $Y$ axis is pointed to the geographical south, and $X$ axis is pointed to the geographical west

(2) $O_{a} X_{a} Y_{a} Z_{a}$ : the azimuth local coordinate connected with the azimuth axis, where the coordinate origin is located on the center of the azimuth track, the $Z_{a}$ axis is coincident with the azimuth axis, and $X_{a}$ and $Y_{a}$ axes are coincident with $X$ and $Y$ axes in the global coordinate initially. When the azimuth axis rotates, $X_{a}$ and $Y_{a}$ axes will also rotate correspondingly, which means the azimuth angle will increase

(3) $O_{e} X_{e} Y_{e} Z_{e}$ : the elevation local coordinate connected with the elevation axis, where the coordinate origin is located on the center of the elevation axis, the $X_{e}$ is coincident with the elevation axis, and $Z_{e}$ is perpendicular to the elevation axis. When the elevation 
axis rotates, all the coordinate axis in this local coordinate will follow this rotation

(4) $O_{r} X_{r} Y_{r} Z_{r}$ : the reflector local coordinate connected with the reflector, where the coordinate origin is located on the center of the reflector, and the $Z_{r}$ axis is coincident with the ideal beam pointing direction if no error existed in the system

In the established coordinate system, if no error has been introduced into the system, the direction of the beam pointing in the reflector local coordinate $O_{r} X_{r} Y_{r} Z_{r}$ can be determined when the azimuth is $\mathrm{A}$ and elevation is $\mathrm{E}$ :

$$
\mathbf{D}^{\mathrm{r}}=\mathbf{R}_{\mathrm{e}}^{\mathrm{r}} \mathbf{R}_{\mathrm{a}}^{\mathrm{e}} \mathbf{R}_{\mathrm{g}}^{\mathrm{a}} \mathbf{D}^{\mathrm{g}}
$$

where $\mathrm{D}^{\mathrm{r}}$ is the coordinates of the beam pointing vector, $\mathbf{R}_{\mathbf{e}}^{\mathbf{r}}$ is the transform matrix between the reflector coordinate and the elevation coordinate, $\mathbf{R}_{\mathbf{a}}^{\mathbf{e}}$ is the transform matrix between the elevation coordinate and the azimuth coordinate, $\mathbf{R}_{\mathbf{g}}^{\mathbf{a}}$ is the transform matrix between the azimuth coordinate and the global coordinate, and $\mathrm{D}^{\mathrm{g}}$ is the beam pointing vector in the global coordinate. The details of this matrix can be found as follows:

$$
\begin{aligned}
& \mathbf{D}^{\mathbf{g}}=\left[\begin{array}{l}
x \\
y \\
z
\end{array}\right]=\left[\begin{array}{c}
\cos E \sin A \\
-\cos E \cos A \\
\sin E
\end{array}\right], \\
& \mathbf{R}_{\mathrm{e}}^{\mathbf{r}}=\left[\begin{array}{lll}
1 & 0 & 0 \\
0 & 1 & 0 \\
0 & 0 & 1
\end{array}\right] \\
& \mathbf{R}_{\mathbf{a}}^{\mathbf{e}}=\left[\begin{array}{ccc}
1 & 0 & 0 \\
0 & \cos \left(\frac{\pi}{2}-E\right) & \sin \left(\frac{\pi}{2}-E\right) \\
0 & -\sin \left(\frac{\pi}{2}-E\right) & \cos \left(\frac{\pi}{2}-E\right)
\end{array}\right], \\
& \mathbf{R}_{\mathrm{g}}^{\mathbf{a}}=\left[\begin{array}{ccc}
\cos A & \sin A & 0 \\
-\sin A & \cos A & 0 \\
0 & 0 & 1
\end{array}\right] .
\end{aligned}
$$

2.4. Modelling of the Telescope Pointing Error with Different Numbers of Support Points. Over the last two decades, numerous large radio telescopes have been constructed and been put into operation to meet the increasing demand from the astronomer and astronautics. In China, the Qi Tai Telescope (QTT), which will be the world's largest steerable telescope, has also been planned and will be under construction soon. Even though all these large radio telescopes can weigh up to thousands of tons, their pointing accuracy

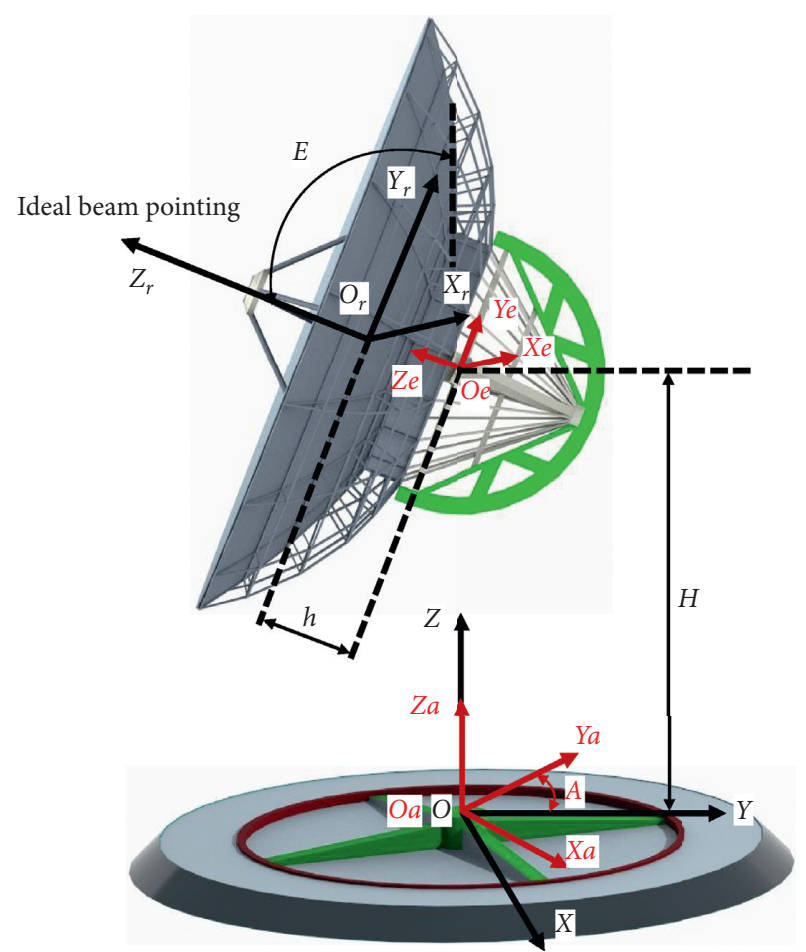

Figure 3: The established coordinates system in the large telescope system.

does not compromise anyway. For example, QTT is designed to work from $150 \mathrm{MHz}$ to $115 \mathrm{GHz}$, which means that the requirement for the pointing accuracy is 1.19 arcsec. Great effort has been made to meet this pointing accuracy requirement, and in this section, the effect of the number of telescope support points on the pointing accuracy has been investigated. Three conceptual design cases have been presented to show this effect.

2.4.1. A Case of the Four-Support-Point Telescope Design. A common practice for a design of telescope support points is using a 4-point wheel design, where the 4-point design constitutes a square as shown in Figure 4. Most of the large telescopes constructed around the world have adopted this type of design, such as the Effelsberg Telescope in Germany and the Green Bank Telescope in America. In this case, the whole telescope structure has been designed to be supported by the four support points, which are in contact with the circular track. The positions of the four support points in the OXYZ coordinate are $(A+(\pi / 4)), \quad(A+(3 \pi / 4))$, $(A+(5 \pi / 4))$, and $(A+(7 \pi / 4))$, and therefore, the track profile amplitude in these positions can be calculated using (1), represented as $f(A+(\pi / 4)), \quad f(A+(3 \pi / 4))$, $f(A+(5 \pi / 4)), f(A+(7 \pi / 4))$ correspondingly. Based on the analysis in [25], the height difference between support point 1 and support point 3 can result in the deviation from the ideal $X$ axis and $Y$ axis, respectively, and the height difference between support point 2 and support point 4 can be obtained by the same method. They can be calculated as 


$$
\left\{\begin{array}{l}
\Delta x_{i}=\left[\frac{f[A+(2 i-1)(\pi / 4)]-f[A+(2 i+3)(\pi / 4)]}{2 r}\right] \cos \left[(2 i-1) \frac{\pi}{4}\right] \\
\Delta \mathbf{y}_{i}=-\left[\frac{f[A+(2 i-1)(\pi / 4)]-f[A+(2 i+3)(\pi / 4)]}{2 r}\right] \cos \left[(2 i-1) \frac{\pi}{4}\right]
\end{array} \quad(i=1,2),\right.
$$

where $i$ represents the support points of the group $i$ with respect to azimuth-axial symmetric distribution, $i=1$ represents support point 1 and support point 3 , and $i=2$ represents support point 2 and support point 4 .

Under the combined action of these four support points, according to the right-hand rule, the pitch axis is rotated by $\varphi_{t x}$ and $\varphi_{t y}$ along the axis of $X_{a}$ and $Y_{a}$, respectively, as in the following:

$$
\begin{aligned}
& \phi_{t x}=\Delta x_{1}+\Delta x_{2}, \\
& \phi_{t y}=\Delta y_{1}+\Delta y_{2} .
\end{aligned}
$$

The rail unevenness can also lead to the stress deformation of the azimuth frame and, thereby, the torsion of the azimuth frame, which makes the pitch rotate around the axis of $Z_{a}$. The displacements of the upper end-points on the pitch axis induced by these support points are denoted as

$$
\begin{aligned}
\varphi_{t z} & =\frac{\left(\delta_{14}-\delta_{23}\right)}{l_{4}}, \\
l_{4} & =2 r \cos \left(\frac{\pi}{4}\right), \\
\delta_{14} & =H\left[\frac{-f(A+(\pi / 4))+f(A+(7 \pi / 4))}{2 r \cos (\pi / 4)}\right], \\
\delta_{23} & =H\left[\frac{-f(A+(3 \pi / 4))+f(A+(5 \pi / 4))}{2 r \cos (\pi / 4)}\right] .
\end{aligned}
$$

As a result, the equations from (4) to (8) can be rewritten in the matrix form, and the large radio telescope pointing error in a design of four support points caused by the rail unevenness can be calculated as

$$
\begin{aligned}
& {\left[\begin{array}{c}
\Delta A \\
\Delta E
\end{array}\right]=\mathbf{C P}_{\mathbf{A}}^{4} \mathbf{N}_{\mathbf{A}}^{4} \text {, }} \\
& \mathbf{C}=\left[\begin{array}{ccc}
0 & \tan E & -1 \\
-1 & 0 & 0
\end{array}\right] \text {, } \\
& \mathbf{P}_{\mathbf{A}}^{4}=\left[\begin{array}{ccccc}
\frac{1}{2 \sqrt{2} r} & -\frac{1}{2 \sqrt{2} r} & -\frac{1}{2 \sqrt{2} r} & \frac{1}{2 \sqrt{2} r} \\
-\frac{1}{2 \sqrt{2} r} & -\frac{1}{2 \sqrt{2} r} & \frac{1}{2 \sqrt{2} r} & \frac{1}{2 \sqrt{2} r} \\
-\frac{H}{2 r^{2}} & \frac{H}{2 r^{2}} & -\frac{H}{2 r^{2}} & \frac{H}{2 r^{2}}
\end{array}\right]=\left[\begin{array}{cccc}
\frac{\cos (\pi / 4)}{2 r} & \frac{\cos (3 \pi / 4)}{2 r} & -\frac{\cos (\pi / 4)}{2 r} & -\frac{\cos (3 \pi / 4)}{2 r} \\
-\frac{\sin (\pi / 4)}{2 r} & -\frac{\sin (3 \pi / 4)}{2 r} & \frac{\sin (\pi / 4)}{2 r} & \frac{\sin (3 \pi / 4)}{2 r} \\
-\frac{H}{\sqrt{4} r^{2}} & \frac{H}{\sqrt{4} r^{2}} & -\frac{H}{\sqrt{4} r^{2}} & \frac{H}{\sqrt{4} r^{2}}
\end{array}\right], \\
& \mathbf{N}_{\mathbf{A}}^{4}=\left[f\left(A+\frac{\pi}{4}\right), f\left(A+\frac{3 \pi}{4}\right), f\left(A+\frac{5 \pi}{4}\right), f\left(A+\frac{7 \pi}{4}\right)\right]^{T},
\end{aligned}
$$

where $\Delta A$ and $\Delta E$ are the pointing error in the azimuth direction and elevation direction. respectively, $\mathrm{C}$ is the elevation position matrix, $\mathbf{P}_{\mathrm{A}}^{4}$ is the azimuth position matrix for a four-support-point design, and $\mathbf{N}_{\mathbf{A}}^{4}$ is the azimuth profile matrix for a four-support-point design. The parameter $r$ is the circular track radius and parameter $H$ is the height difference between the elevation axis and the ground. The detailed process on how to deduce this relationship can be found in $[16,25]$.
2.4.2. A Case of a Six-Support-Point Telescope Design. In the previous papers, only a four-support-point case has been considered to study the effect of the rail unevenness on the pointing accuracy of the large radio telescope. However, there is no direct evidence to show that the four-supportpoint support design is the best from the point of view of increasing the telescope pointing accuracy. For example, Tianma (TM) Telescope built in 2012 has adopted a sixsupport-point design to support the whole telescope 


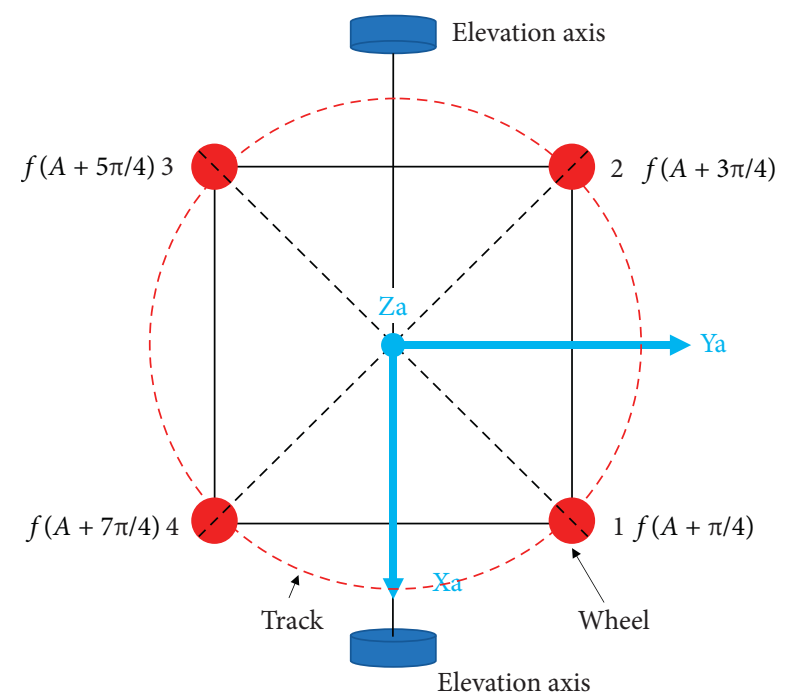

FIgURE 4: The sketch of the four-support-point telescope design.

structure, and the overall pointing accuracy for TM telescope still can achieve 12.36". In the following section, a six-support-point design has been proposed to investigate the effect of telescope azimuth track unevenness on the pointing accuracy. The positions of the six support points in the OXYZ coordinate are $(A+(\pi / 6)),(A+(3 \pi / 6))$, $(A+(5 \pi / 6)), \quad(A+(7 \pi / 6)), \quad(A+(9 \pi / 6)), \quad$ and

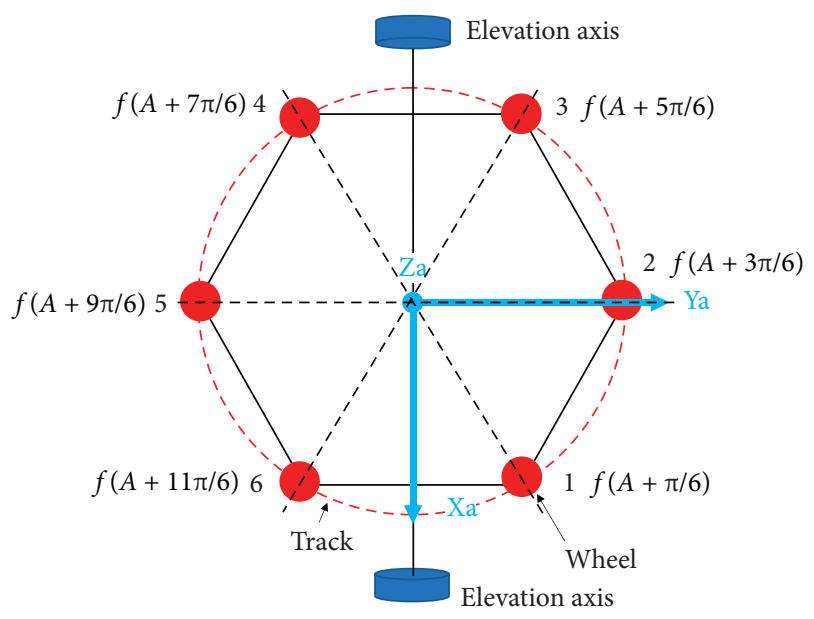

Figure 5: The sketch of the six support points telescope design.

$(A+(11 \pi / 6))$, respectively, and therefore, the track profile amplitude in these positions can be represented as $f(A+(\pi / 6)), f(A+(3 \pi / 6)), \mathrm{f}(\mathrm{A}+(5 \pi / 6)), f(A+(7 \pi / 6)$ ),$f(A+(9 \pi / 6))$, and $f(A+(11 \pi / 6))$ accordingly. The detailed information can be found in Figure 5. Based on the analysis in [25], the height difference between support point $i(i=1,2,3)$ and support point $i+3$ can result in the deviation from the ideal $X$ axis and $Y$ axis, respectively, and they can be calculated as

$$
\left\{\begin{array}{l}
\Delta x_{i}=\left[\frac{f[A+(2 i-1)(\pi / 6)]-f[A+(2 i+5)(\pi / 6)]}{2 r}\right] \cos [(2 i-1)(\pi / 6)], \\
\Delta \mathbf{y}_{i}=-\left[\frac{f[A+(2 i-1)(\pi / 6)]-f[A+(2 i+5)(\pi / 6)]}{2 r}\right] \cos [(2 i-1)(\pi / 6)],
\end{array} \quad(i=1,2,3),\right.
$$

where $i$ represents the support points of the group $i$. The grouping of the six support points is similar to that of the four support points; that is, the support points related to azimuth-axial symmetry are grouped into a group.

Under the combined action of six points, according to the right-hand rule, the pitch axis is rotated by $\varphi_{t x}$ and $\varphi_{t y}$ along the axis of $X_{a}$ and $Y_{a}$, respectively, as in the following:

$$
\begin{aligned}
& \phi_{t x}=\Delta x_{1}+\Delta x_{2}+\Delta x_{3}, \\
& \phi_{t y}=\Delta y_{1}+\Delta y_{2}+\Delta y_{3} .
\end{aligned}
$$

In addition, the rail unevenness can also lead to the stress deformation of the azimuth frame and, thereby, the torsion of the azimuth frame, which makes the pitch rotate around the axis of $Z_{a}$. The displacements of the upper end-points on the pitch axis induced by these support points are denoted as

$$
\begin{aligned}
& \varphi_{t z}=\frac{\left(\delta_{16}-\delta_{25}\right)}{l_{6}}-\frac{\left(\delta_{25}-\delta_{34}\right)}{l_{6}}, \\
& l_{6}=2 r \cos \left(\frac{\pi}{6}\right), \\
& \delta_{16}=H\left[\frac{-f(A+(\pi / 6))+f(A+(11 \pi / 6))}{r}\right], \\
& \delta_{25}=H\left[\frac{-f(A+(3 \pi / 6))+f(A+(9 \pi / 6))}{2 r}\right], \\
& \delta_{34}=H\left[\frac{-f(A+(5 \pi / 6))+f(A+(7 \pi / 6))}{r}\right] .
\end{aligned}
$$

The equations from (11) to (14) can be rewritten in the matrix form as 


$$
\begin{aligned}
& {\left[\begin{array}{l}
\phi_{t x} \\
\phi_{t y} \\
\phi_{t z}
\end{array}\right]=\mathbf{P}_{\mathbf{A}}^{6} \mathbf{N}_{\mathbf{A}}^{6} \text {, }} \\
& \mathbf{P}_{\mathbf{A}}^{6}=\left[\begin{array}{cccccc}
\frac{\cos (\pi / 6)}{2 r} & \frac{\cos (3 \pi / 6)}{2 r} & \frac{\cos (5 \pi / 6)}{2 r} & -\frac{\cos (\pi / 6)}{2 r} & -\frac{\cos (3 \pi / 6)}{2 r} & -\frac{\cos (5 \pi / 6)}{2 r} \\
-\frac{\sin (\pi / 6)}{2 r} & -\frac{\sin (3 \pi / 6)}{2 r} & -\frac{\sin (5 \pi / 6)}{2 r} & \frac{\sin (\pi / 6)}{2 r} & \frac{\sin (3 \pi / 6)}{2 r} & \frac{\sin (5 \pi / 6)}{2 r} \\
-\frac{H}{\sqrt{3} r^{2}} & \frac{H}{\sqrt{3} r^{2}} & -\frac{H}{\sqrt{3} r^{2}} & \frac{H}{\sqrt{3} r^{2}} & -\frac{H}{\sqrt{3} r^{2}} & \frac{H}{\sqrt{3} r^{2}}
\end{array}\right], \\
& \mathbf{N}_{\mathbf{A}}^{6}=\left[f\left(A+\frac{\pi}{6}\right) f\left(A+\frac{3 \pi}{6}\right) f\left(A+\frac{5 \pi}{6}\right) f\left(A+\frac{7 \pi}{6}\right) f\left(A+\frac{9 \pi}{6}\right) f\left(A+\frac{11 \pi}{6}\right)\right]^{T} .
\end{aligned}
$$

Similar to (4), the large radio telescope pointing error in a six-support-point design caused by the rail unevenness can be calculated as

$$
\left[\begin{array}{c}
\Delta A \\
\Delta E
\end{array}\right]=\mathbf{C P}_{\mathbf{A}}^{6} \mathbf{N}_{\mathbf{A}}^{6} \text {, }
$$

where $C$ is still the elevation position matrix, as shown in (4), $i=2$, while $\mathbf{P}_{\mathbf{A}}^{6}$ is the azimuth position matrix for a sixsupport-point design and $\mathbf{N}_{\mathbf{A}}^{6}$ is the azimuth profile matrix for a six-support-point design, which all have been given in (10) and (11) respectively.

2.4.3. A Case of an Eight-Support-Point Telescope Design. In this case, the boundary on the number of the large radio telescope support points has been further pushed forward and an eight-support-point concept for the large radio telescope has been investigated in this section, as shown in Figure 6 . The positions of the eight support points in the OXYZ coordinate can be determined as $(A+(\pi / 8))$, $(A+(3 \pi / 8)), \quad(A+(5 \pi / 8)), \quad(A+(7 \pi / 8)), \quad(A+(9 \pi / 8))$, $(A+(11 \pi / 8)),(A+(13 \pi / 8))$, and $(A+(15 \pi / 8))$, and the track profile amplitude in these positions can be represented as $f(A+(\pi / 8)), f(A+(3 \pi / 8)), f(A+(5 \pi / 8)), f(A+(7 \pi$ /8) $), f(A+(9 \pi / 8)), f(A+(11 \pi / 8)), f(A+(13 \pi / 8))$, and $f(A+(15 \pi / 8))$ accordingly. Similar to the analysis in a sixsupport-points design, the height difference between support point $i(i=1,2,3,4)$ and support point $i+4$ can result in the deviation from the ideal $X$ axis and $Y$ axis, respectively, and they can be calculated as

$$
\left\{\begin{array}{l}
\Delta x_{i}=\left[\frac{f[A+(2 i-1)(\pi / 8)]-f[A+(2 i+7)(\pi / 8)]}{2 r}\right] \cos [(2 i-1)(\pi / 8)], \\
\Delta \mathbf{y}_{i}=-\left[\frac{f[A+(2 i-1)(\pi / 8)]-f[A+(2 i+7)(\pi / 8)]}{2 r}\right] \cos [(2 i-1)(\pi / 8)],
\end{array} \quad(i=1,2,3,4) .\right.
$$

The grouping of the eight support points is similar to the previous grouping of the four support points in (a) and the six support points in (b) and will not be repeated here.

Under the combined action of six points, according to the right-hand rule, the pitch axis is rotated by $\varphi_{t x}$ and $\varphi_{t y}$ along the axis of $X_{a}$ and $Y_{a}$, respectively, as in the following:

$$
\begin{aligned}
& \phi_{t x}=\Delta x_{1}+\Delta x_{2}+\Delta x_{3}+\Delta x_{4}, \\
& \phi_{t y}=\Delta y_{1}+\Delta y_{2}+\Delta y_{3}+\Delta y_{4} .
\end{aligned}
$$

In addition, the rail unevenness can also lead to the stress deformation of the azimuth frame and, thereby, the torsion of the azimuth frame, which makes the pitch rotate around the axis of $Z_{a}$. The displacements of the upper endpoints on the pitch axis induced by these support points are denoted as

$$
\begin{aligned}
\varphi_{t z} & =\frac{\left(\delta_{18}-\delta_{45}\right)}{l_{81}}-\frac{\left(\delta_{27}-\delta_{36}\right)}{l_{82}}, \\
l_{81} & =2 r \sin \left(\frac{\pi}{8}\right) \\
l_{82} & =2 r \cos \left(\frac{\pi}{8}\right)
\end{aligned}
$$




$$
\begin{aligned}
& \delta_{18}=H\left[\frac{-f(A+(\pi / 8))+f(A+(15 \pi / 8))}{2 r \sin (\pi / 8)}\right], \\
& \delta_{27}=H\left[\frac{-f(A+(3 \pi / 8))+f(A+(13 \pi / 8))}{2 r \cos (\pi / 8)}\right], \\
& \delta_{36}=H\left[\frac{-f(A+(5 \pi / 8))+f(A+(11 \pi / 8))}{2 r \cos (\pi / 8)}\right], \\
& \delta_{45}=H\left[\frac{-f(A+(7 \pi / 8))+f(A+(9 \pi / 8))}{2 r \sin (\pi / 8)}\right] .
\end{aligned}
$$

$$
\begin{aligned}
& {\left[\begin{array}{c}
\phi_{t x} \\
\phi_{t y} \\
\phi_{t z}
\end{array}\right]=\mathbf{P}_{\mathbf{A}}^{8} \mathbf{N}_{\mathbf{A}}^{8},}
\end{aligned}
$$

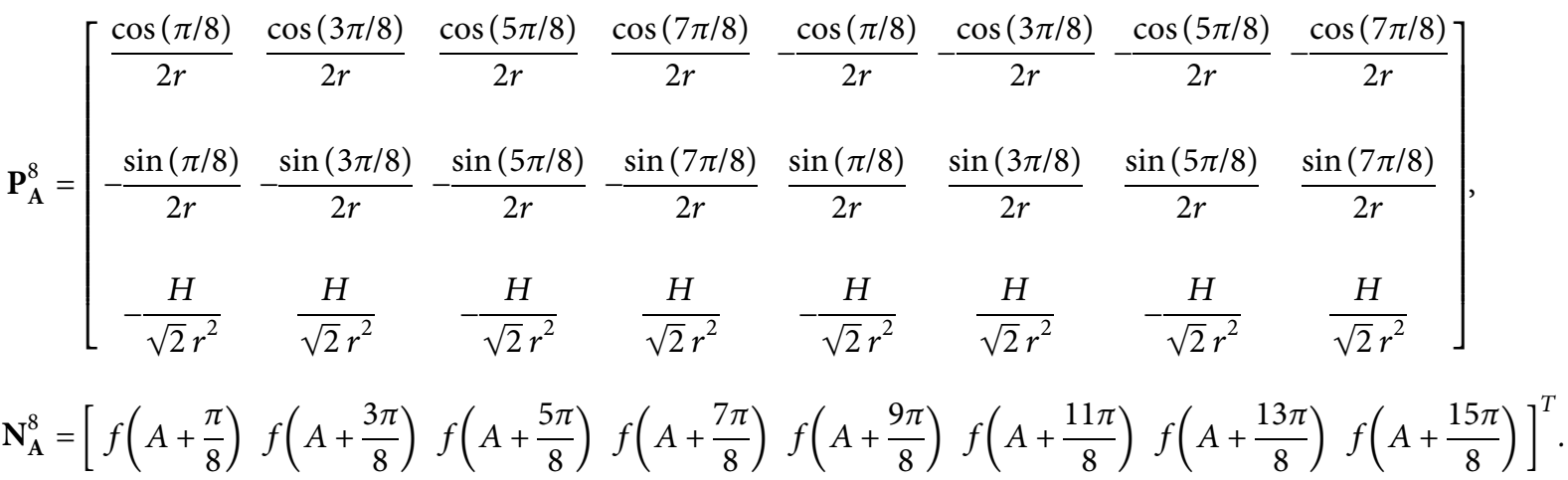

Similar to (4) and (13), the large radio telescope pointing error in a six-support-point design caused by the rail unevenness can be calculated as

$$
\left[\begin{array}{c}
\Delta A \\
\Delta E
\end{array}\right]=\mathbf{C P}_{\mathbf{A}}^{8} \mathbf{N}_{\mathbf{A}}^{8} \text {, }
$$

where $\mathbf{C}$ is still the elevation position matrix, as shown in (4), $i=2$, while $\mathbf{P}_{\mathbf{A}}^{8}$ is the azimuth position matrix for an eightsupport-point design and $\mathbf{N}_{\mathbf{A}}^{8}$ is the azimuth profile matrix for an eight-support-point design, which all have been given in (17) and (18), respectively.

\section{Numerical Investigation of the Pointing Accuracy in the Telescope System}

A numerical process for evaluating the effect of the azimuth track unevenness on a large radio telescope pointing accuracy has been developed in this research, as shown in Figure 7. First, the number of sampling points can be determined based on the azimuth track dimension, and then the track profile raw data can be measured correspondingly by the leveling instrument. In [12], a sampling frequency of
The equations from (17) to (21) can be rewritten in the matrix form as
$2 \mathrm{~Hz}$ with a constant rotating rate of $50 \mathrm{~m} \cdot \mathrm{deg} / \mathrm{s}$ was used taking into consideration the measuring noise level, temperature gradient impact, and so on. The author also suggested that the measurement can be performed during the night time in order to minimize the distortions of the telescope structure due to the thermal gradient. Next, the coordinate systems described in Section 2.3 can be established accordingly, and the azimuth track raw data can then be described in these coordinates, respectively. However, all these sampling data have been discretized, and in order to have a continuous description of the azimuth track profile, the Fourier function equation as shown in (1) can be used to model the azimuth track profile. After that, the azimuth track profile can be written as a mathematical function, and then the following steps will be run until all the pointing errors on every azimuth and elevation angle have been calculated.

(1) Set an initial elevation angle $E(j)$ and the azimuth angle $A(i)$

(2) Calculate the pointing error in both the azimuth direction and the elevation direction using the matrix discussed in Section 2. It should be noted that 


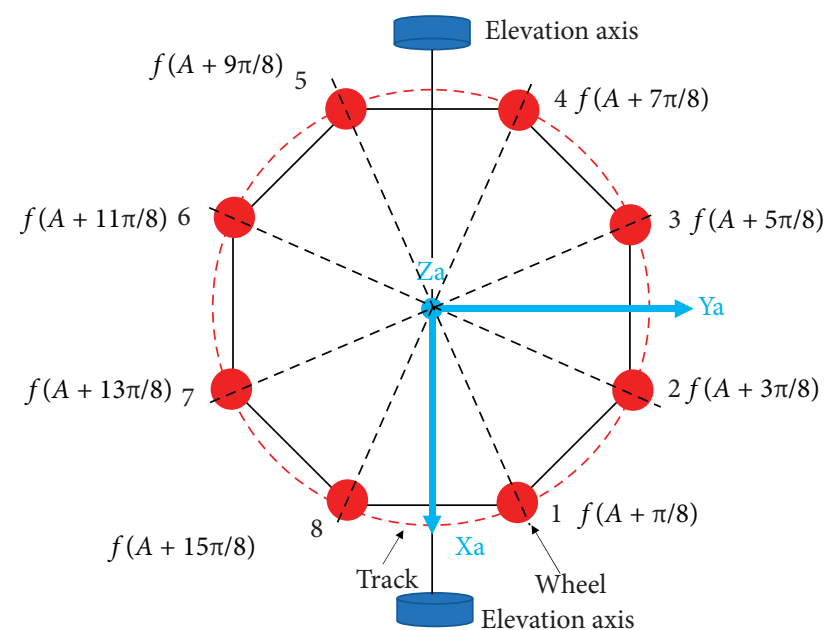

Figure 6: The sketch of the eight-support-point telescope design.

different telescope support point numbers should select different matrices based on the analysis in Section 2

(3) The telescope pointing error at position $E(j)$ and $A(i)$ can be calculated as

$$
\varphi_{i j}=\sqrt{\left[\cos (E) \Delta A z_{i}\right]^{2}+\left(\Delta E l_{j}\right)^{2}} .
$$

(4) The telescope pointing error RMS value can be calculated as

$$
\mathrm{RMS}_{\mathrm{BS}}=\sqrt{\frac{\sum_{i=1}^{M} \sum_{j=1}^{N}\left(\varphi_{i j}\right)^{2}}{M \bullet N}} .
$$

In the end, the large radio telescope pointing error can be estimated for different azimuth track profiles as well as for different telescope support points conditions.

One numerical example has been presented in this research to demonstrate the proposed evaluating process. The example is an azimuth track with a $0.14 \mathrm{~mm}$ azimuth track average value. In total, 120 sampling points along the azimuth track were recorded. The Fourier coefficients for the azimuth track profile can then be determined using the least squared method mentioned in Section 2. A comparison of the fitting curve and the raw data can be found in Figure 8. It can be found that the resultant fitting curve can describe the overall trend of these raw data in good consistency.

Figure 9 shows the telescope pointing error for the four support points case when its elevation angle is set to be $1 \mathrm{deg}$. It is interesting to see that the whole pointing error curve has four similar peaks during one azimuth rotation. This phenomenal is reasonable because there are four support points in the telescope design, and when the telescope rotates in the azimuth direction, support point number 1 will move from position $A$ to position $B$ while, simultaneously, support point number 2 will move from position $B$ to position $C$. The

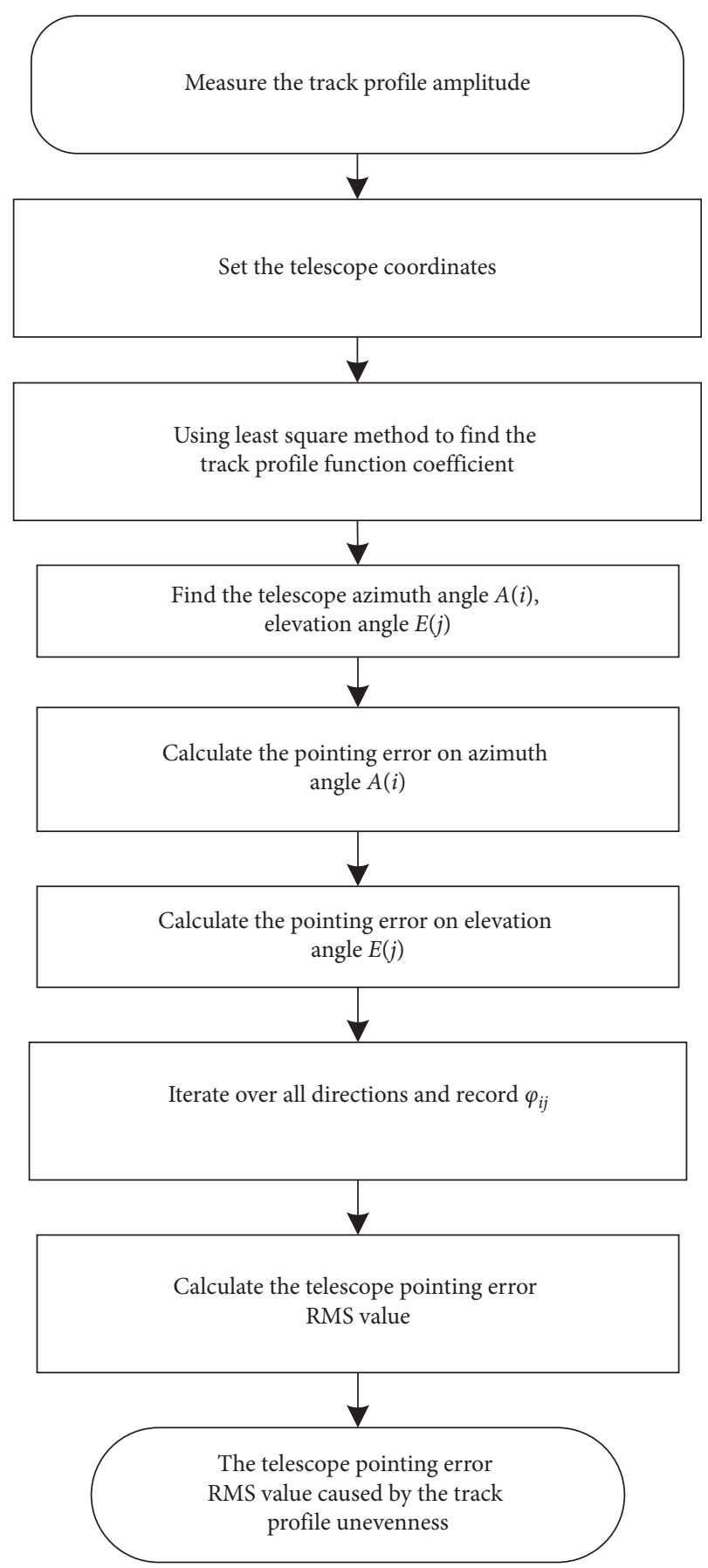

FIgURE 7: The numerical process for evaluating the telescope pointing error.

same situation is with support point number 3 and support point number 4 . In this case, support point number 1 would take position $B$ which is used to be occupied by support point number 2 and, in turn, support point number 2 would take support point number 3's position and so on. If all the support points in the telescope are assumed to be identical, the telescope pointing error would only be related to the azimuth track profile. In other words, the telescope pointing error would only be related to the support-point positions, which is from position $A$ to position $D$. Taking a closer look 


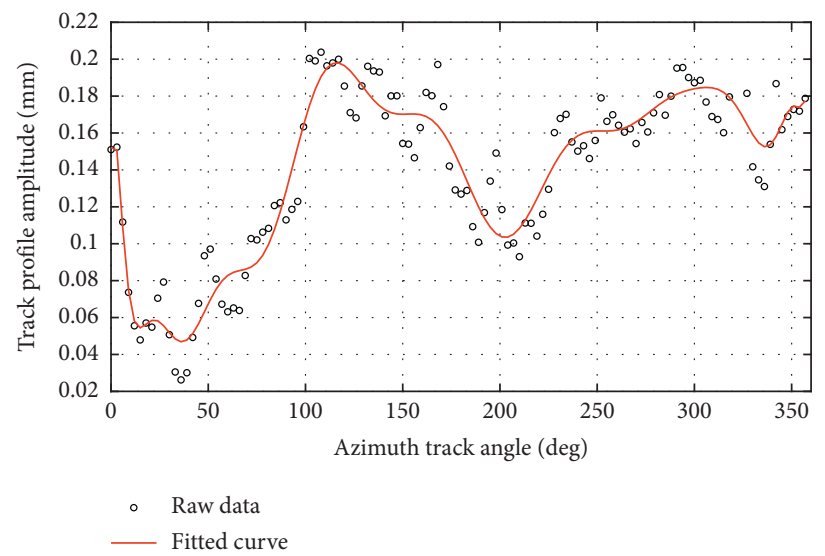

FIgURE 8: The azimuth track profile for the telescope with a $34 \mathrm{~m}$ diameter [24].

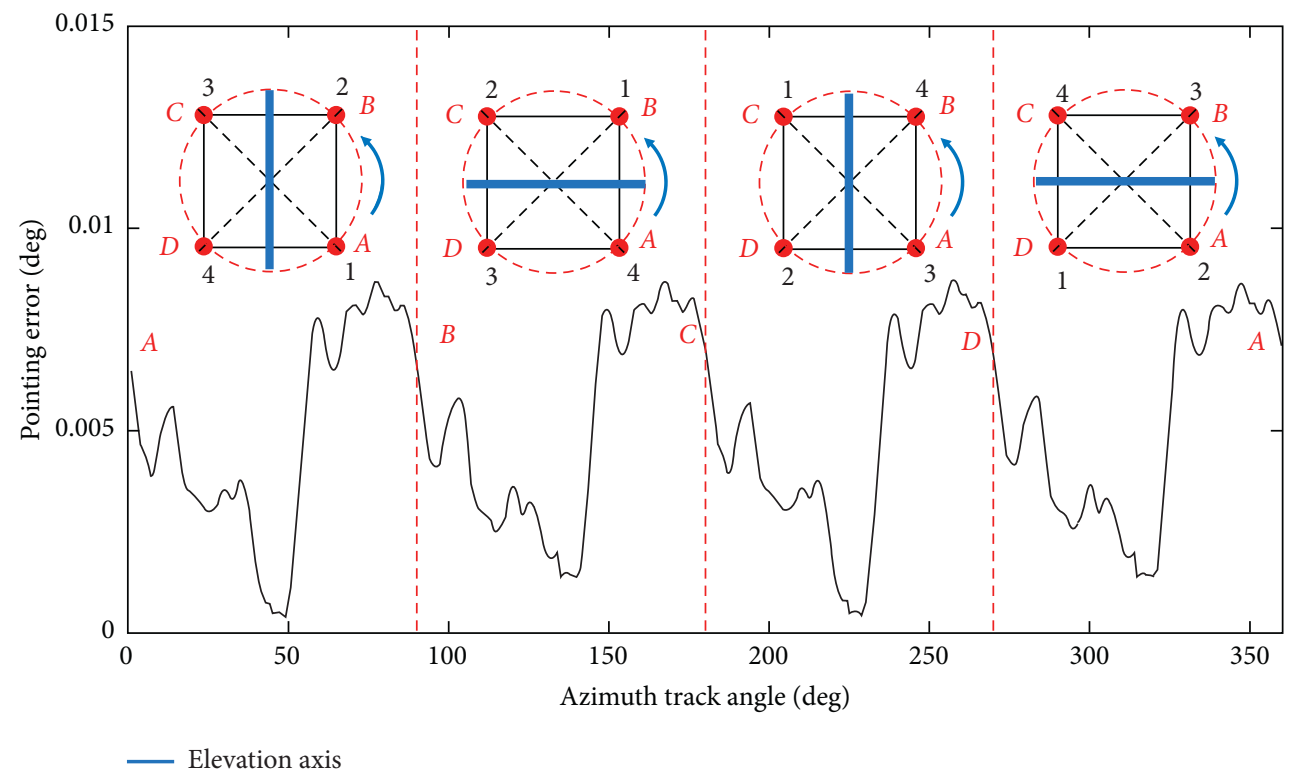

Figure 9: Telescope pointing error for a four-support-point case (Elevation angle is set to be $1 \mathrm{deg}$ ).

at the figure, we can find that the four periods are not exactly identical to each other as a comparison can be made between the curves in the $A B$ range and $B C$ range. This is, largely, because the direction of the elevation axis is different in these two ranges, as shown in the figure. However, this difference is relatively small compared with the overall pointing error value. It should also be pointed out that the pointing error curve in the $A B$ range is the same as that in the $C D$ range while the pointing error curve in the $B C$ range is the same as that in the $D A$ range.

Figure 10 shows the telescope pointing error for the foursupport-point case for the whole scanning range, where the elevation angle ranged from $0 \mathrm{deg}$ to $80 \mathrm{deg}$. In the figure, four dominant ranges can be easily identified as discussed previously. However, one thing should be notified: in the domain of $B C$ and $C D$, the pointing error was observed to have an increasing trend with the rotation of the elevation angle and two obvious peaks in these two domains can be observed when the elevation angle is close to $80 \mathrm{deg}$. By following the procedure presented in Figure 7, the overall RMS value for the telescope pointing error can be calculated to be $0.0044 \mathrm{deg}$ in this case.

Figure 11 shows the telescope pointing error for the sixsupport-point case when its elevation angle is set to be $1 \mathrm{deg}$. Similarly, it is interesting to notice that the whole pointing error curve has six similar peaks during one azimuth rotation, which is corresponding to six support points. This phenomenon can also be explained by the analysis presented in the four-support-point case, where the support point number 1 will take the positions of $A, B, \ldots, F$ successively, and the telescope pointing error curve will repeat itself in the domain $A B$ and $D E$, domain $B C$ and $E F$, and domain $C D$ and $F A$, respectively. A clear view of the explanation can be found in Figure 11, where the direction of the elevation axis in every domain can be clearly identified.

Figure 12 shows the telescope pointing error for the sixsupport-point case for the whole scanning range, where the elevation angle still ranged from 0 deg to $80 \mathrm{deg}$. It should be 


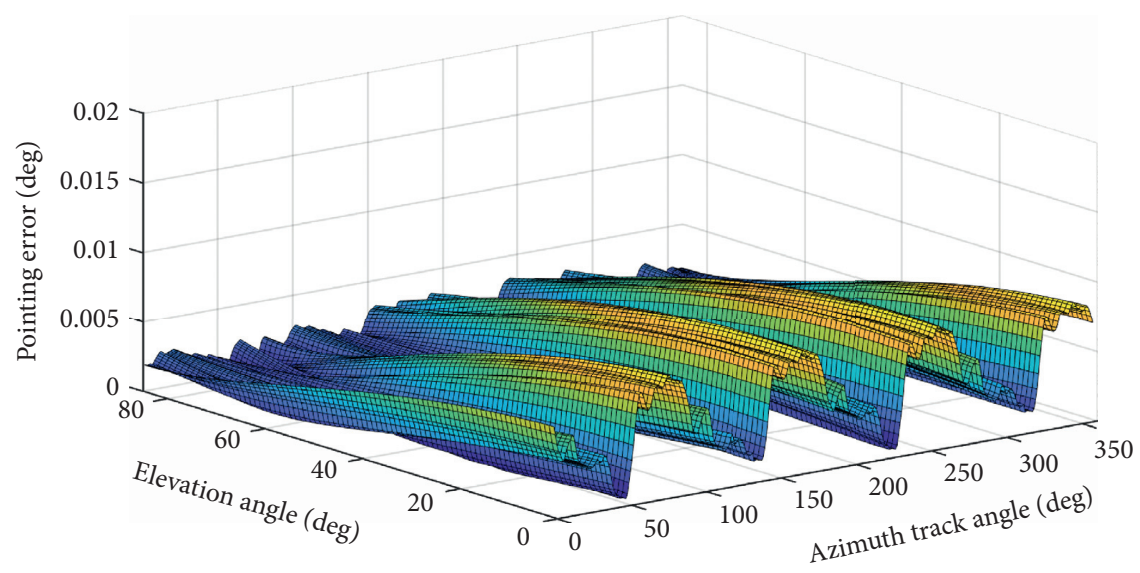

Figure 10: Telescope pointing error for a four-support-point case.

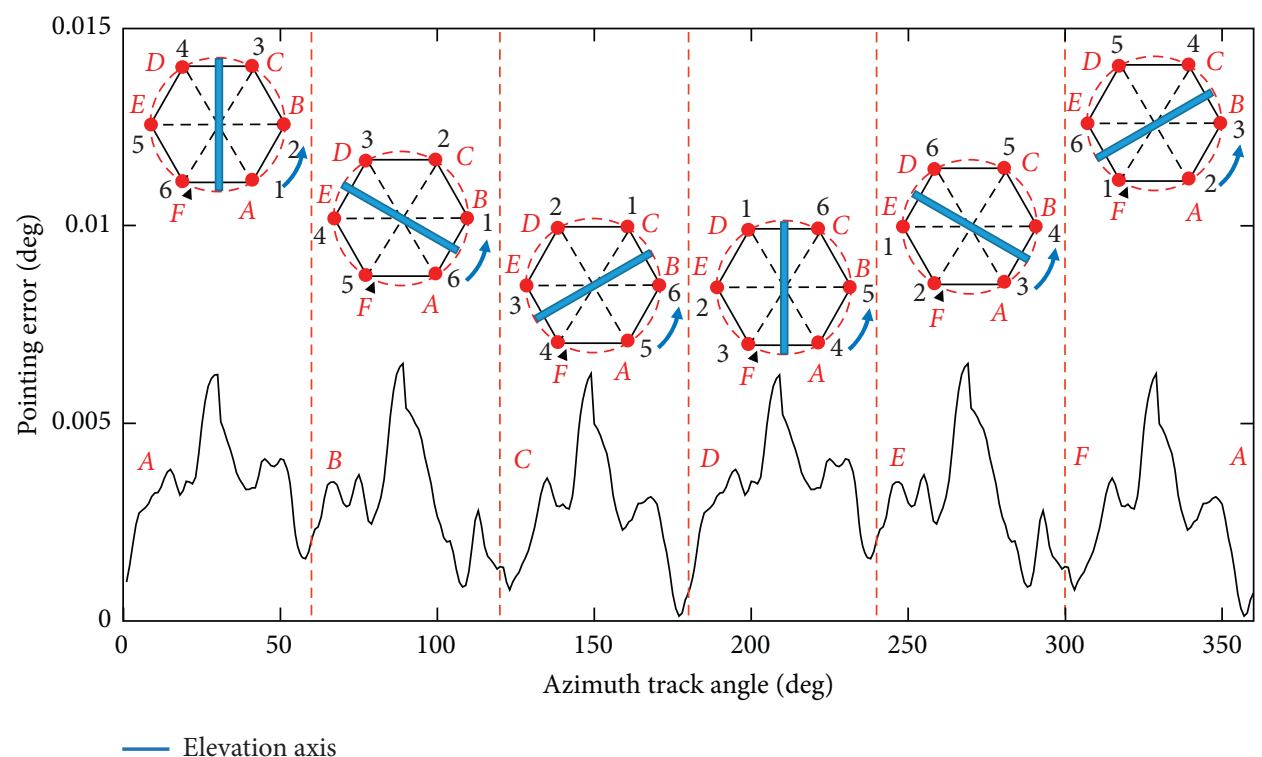

FIgURE 11: Telescope pointing error for the six-support-point case (Elevation angle is set to be $1 \mathrm{deg}$ ).

noticed that the telescope azimuth track profile is the same as the four-support-point case and the only difference is the telescope support-point number. In the figure, six dominant ranges can also be easily identified and the pointing error was observed to have an increasing trend with the rotation of the elevation angle in domain $C D$ and $F A$. By following the procedure presented in Figure 7, the overall RMS value for the telescope pointing error, in this case, can be calculated to be 0.0032 deg.

Figure 13 shows the telescope pointing error for the eight-support-point case when its elevation angle is set to be $1 \mathrm{deg}$. Similarly, it is interesting to notice that the whole pointing error curve has eight similar peaks during one azimuth rotation, which is corresponding to these eight support points. During the rotation, the support point number 1 will take the positions of $A, B, \ldots, H$ in a sequence, and the telescope pointing error curve will repeat itself in four different domains. Three examples, which show the support point positions and elevation axis direction, can be found in the figure.
Figure 14 shows the telescope pointing error for the eight-support-point case for the whole scanning range, where the elevation angle still ranged from 0 deg to $80 \mathrm{deg}$. The telescope azimuth track profile used in the four-supportpoint and six-support-point cases will be adopted here. In the figure, the telescope pointing error fluctuation along the azimuth track rotation and the elevation rotation can be observed; however, it is hard to distinguish each domain in this case. Except for this, the pointing error was still to be observed to have an increasing trend with the rotation of the elevation angle, which is similar to the four-support-points case as well as the six-support-point case. The overall RMS value for the telescope pointing error, in this case, can be calculated to be $0.0046 \mathrm{deg}$.

Figure 15 presents a summary of telescope pointing error statistic parameters for different support point number cases. The most important statistic parameter for the pointing error is the root means square (RMS) value, whose calculation detail can be found in Figure 7. As shown in the figure, the blue bar stands for the RMS value and it is 


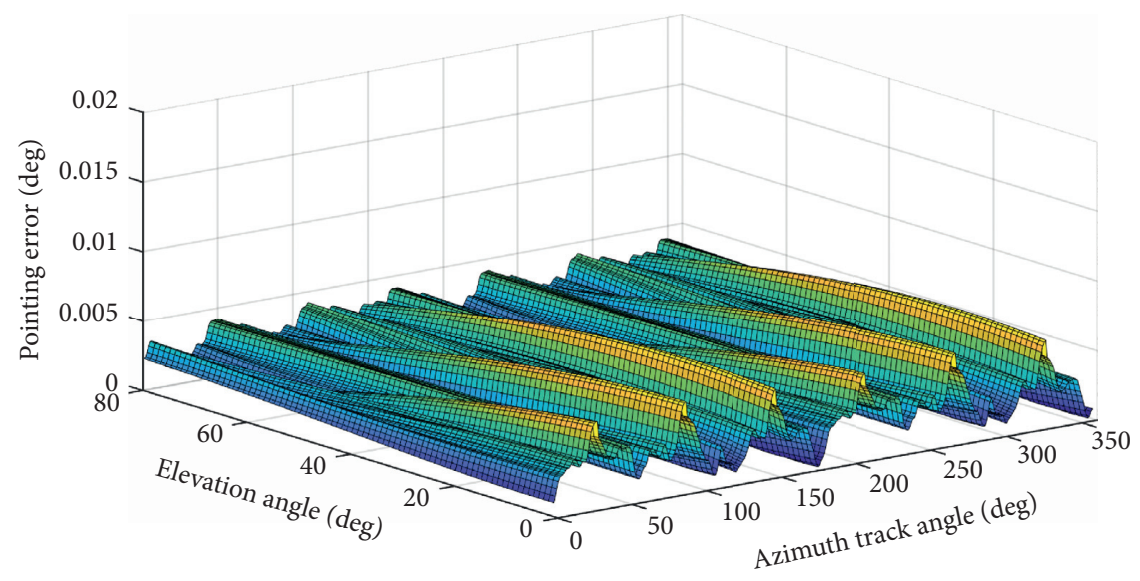

FIGURE 12: Telescope pointing error for the six-support-point case.

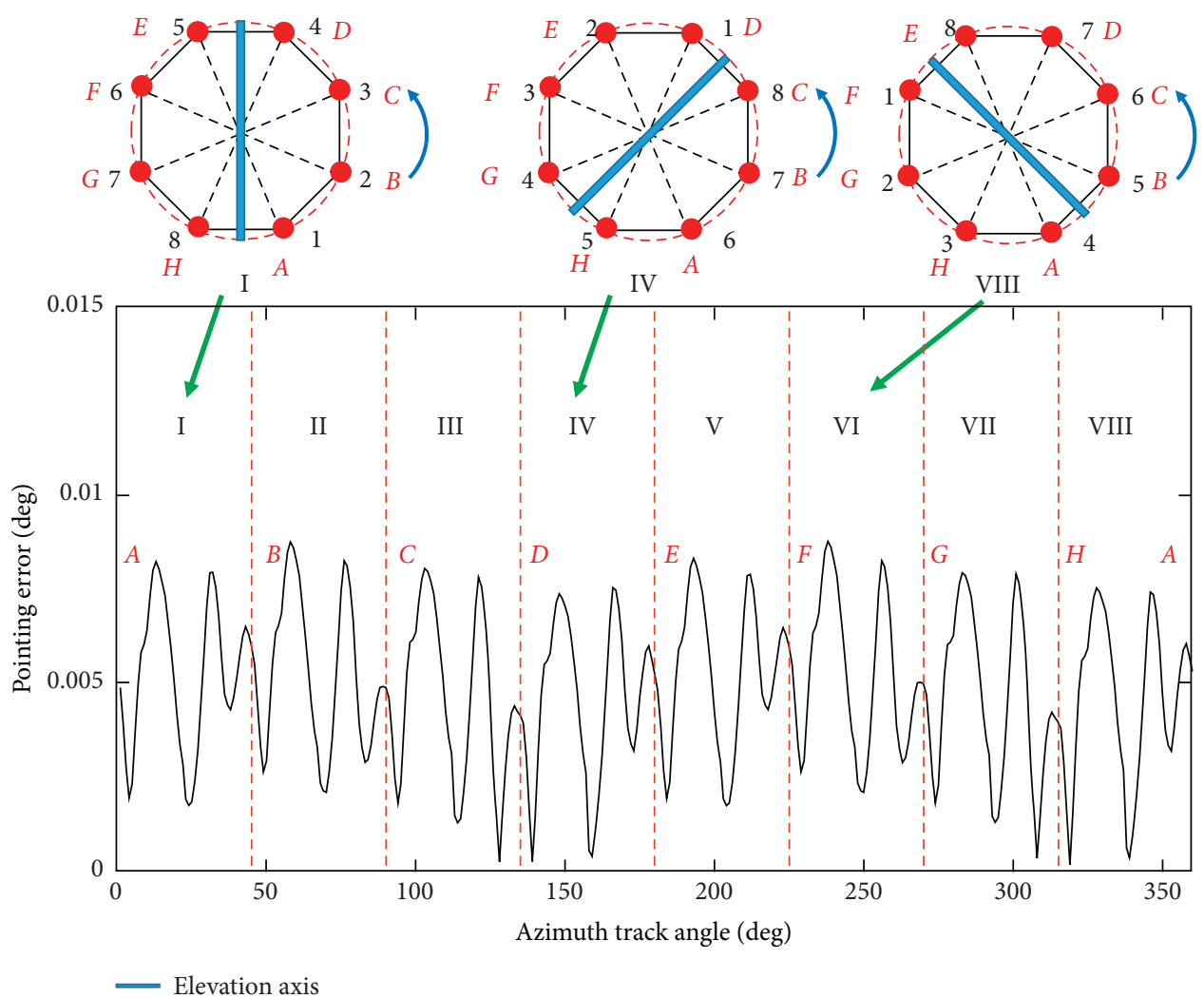

FIgURE 13: Telescope pointing error for the eight-support-point case (Elevation angle is set to be $1 \mathrm{deg}$ ).

interesting to see that there is not a monotonous relationship between the RMS value and the telescope support point number. In detail, the RMS value for the telescope foursupport-point case is around $0.0044 \mathrm{deg}$, which is larger than the value for the six-support-point case. There is a $0.0012 \mathrm{deg}$ reduction in the six-support-point case. However, the RMS value increases to $0.0046 \mathrm{deg}$ in the eight-support-point case, which suggests simply that increasing the telescope number will not decrease the RMS value for sure. A similar trend can be found for the standard deviation value and the mean value, where the six-support-point case is the optimal choice in terms of improving the telescope pointing error value.

\section{Discussion}

This paper has discussed the impact of different telescope support point numbers on the pointing accuracy as the most dominant practice for the telescope design is to use a foursupport-point design, and currently, there is no further study to investigate the effectiveness of other telescope designs in terms of the support point numbers. However, there were several major assumptions in this research. Firstly, the Fourier function was used in this research to estimate the measured raw data. In [16], a coarse-fine mixed model was proposed to describe the track unevenness. Even though a smaller 


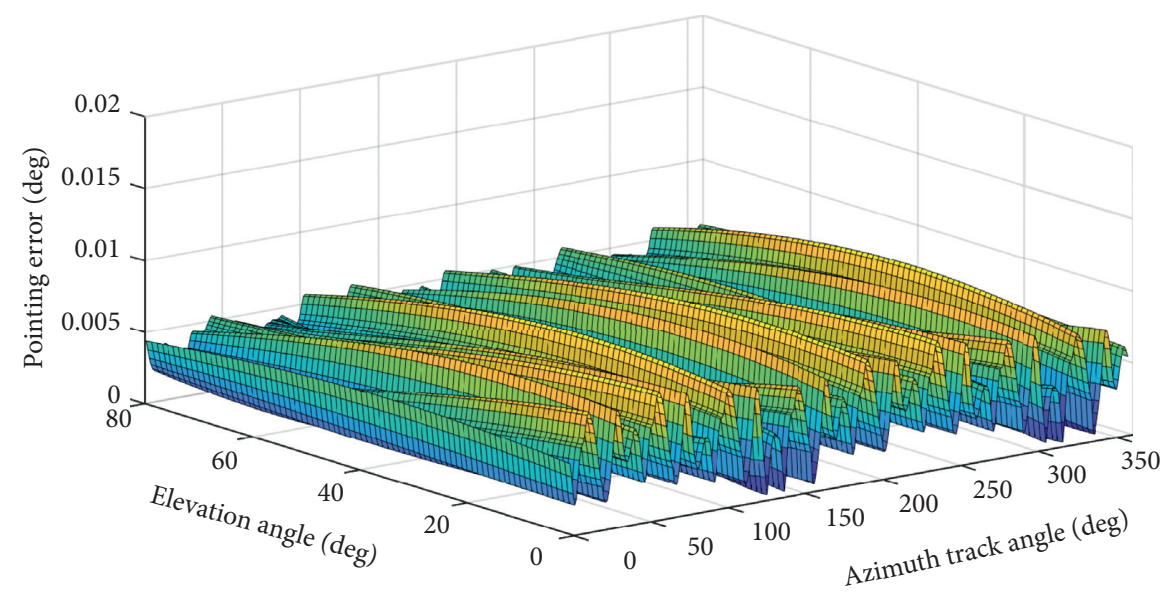

FIGURE 14: Telescope pointing error for the eight-support-point case.

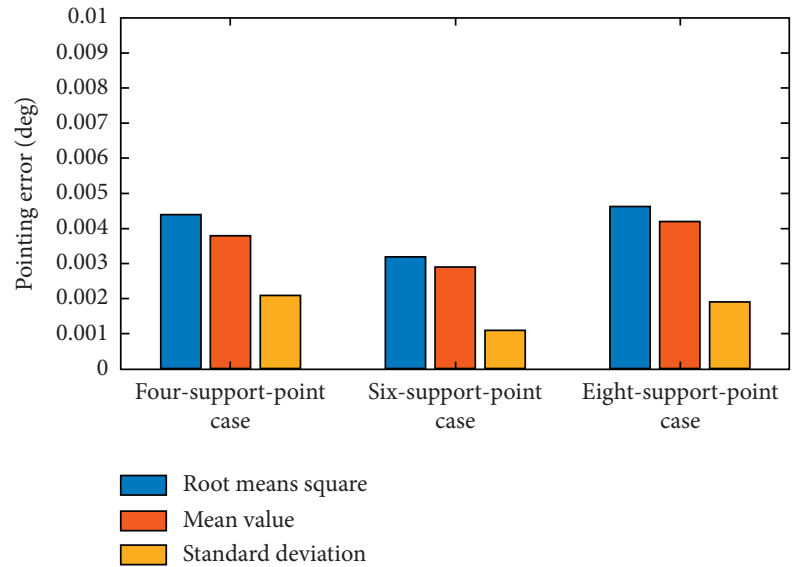

Figure 15: Summary of telescope pointing error statistics parameters.

estimation error was reported in their study, more unknown parameters were required during the estimation process. The Fourier fitting adopted in this research is a compromise result between the accuracy and the unknown parameter number, while it can still characterize the main trend of the measured data. Secondly, the profile of the telescope's wheel is not considered during the estimation. Actually, the consideration of the profile interaction between the telescope support points and the azimuth track will definitely change the results presented in this research. However, the telescope's wheel is assumed to be identical in this research, and the profile interaction between the wheel and azimuth track will be considered in the future. Thirdly, the placement of the elevation axis is set to be in the middle of the two support points. Other elevation axis directions can also be selected, but the proposed azimuth position matrix $\mathrm{P}_{\mathrm{A}}$ will be different, and consequently, the estimated telescope pointing error will be different.

In this paper, a general telescope pointing error estimation formula for different support point numbers can be derived and its matrix form can be given as

$$
\left[\begin{array}{c}
\Delta A \\
\Delta E
\end{array}\right]=\mathbf{C P}_{\mathbf{A}}^{\mathbf{i}} \mathbf{N}_{\mathbf{A}}^{\mathbf{i}} \text {. }
$$

In the equation, $\mathrm{C}$ is the elevation position matrix, which is a function of the elevation angle. This means that the matrix $\mathbf{C}$ is the same for all the cases. Matrix $\mathbf{P}_{\mathbf{A}}^{\mathbf{i}}$ is the azimuth position matrix for a design with $\boldsymbol{i}$ support points. This matrix is a function of the support-point positions, and the details on how to construct this matrix can be found in [25] and in Section 2. Matrix $\mathbf{N}_{\mathbf{A}}^{\mathbf{i}}$ is the azimuth profile matrix for a design with i support points. Actually, this matrix is only used to extract the track profile amplitude on the positions where the support points are located. When the track profile and the telescope support-point positions are determined, this matrix can be determined from the fitting function. All in all, this general formula provides a quick estimation tool for the telescope pointing accuracy with different support point numbers, and the most difficult part in this formula is the construction of the matrix $\mathbf{P}_{\mathbf{A}}^{\mathbf{i}}$, which is different for different support point designs.

Figures 9, 11, and 13 give the telescope pointing error for a fixed elevation angle during the whole azimuth track rotation for the four-support-point case, six-support-point case, and eight-support-point case, respectively. All these figures suggested that the telescope support point numbers can have a huge impact on the pointing error curve characteristics, which can have corresponding dominant peaks. This phenomenon can be explained by the fact that the support points will occupy the same azimuth track positions consequently during one azimuth rotation, and if all the wheels in the telescope are assumed to be identical, the telescope pointing error peak would repeat itself the number of times, which the telescope support point numbers have. Figures 10, 12, and 14 give the telescope pointing error distribution during the whole azimuth track rotation as well as the half elevation rotation for the foursupport-point case, six-support-point case, and eight-supportpoint case, respectively. The RMS value for this pointing error distribution can be performed, and a summary of the results can be found in Figure 15. It is interesting to find that the RMS value experienced a complex trend with the support point number change, and there is not a simple monotonous increasing or decreasing relationship. This is largely caused by the strong coupling effect between each support point in the azimuth position matrix $\mathbf{P}_{\mathbf{A}}^{\mathbf{i}}$. 


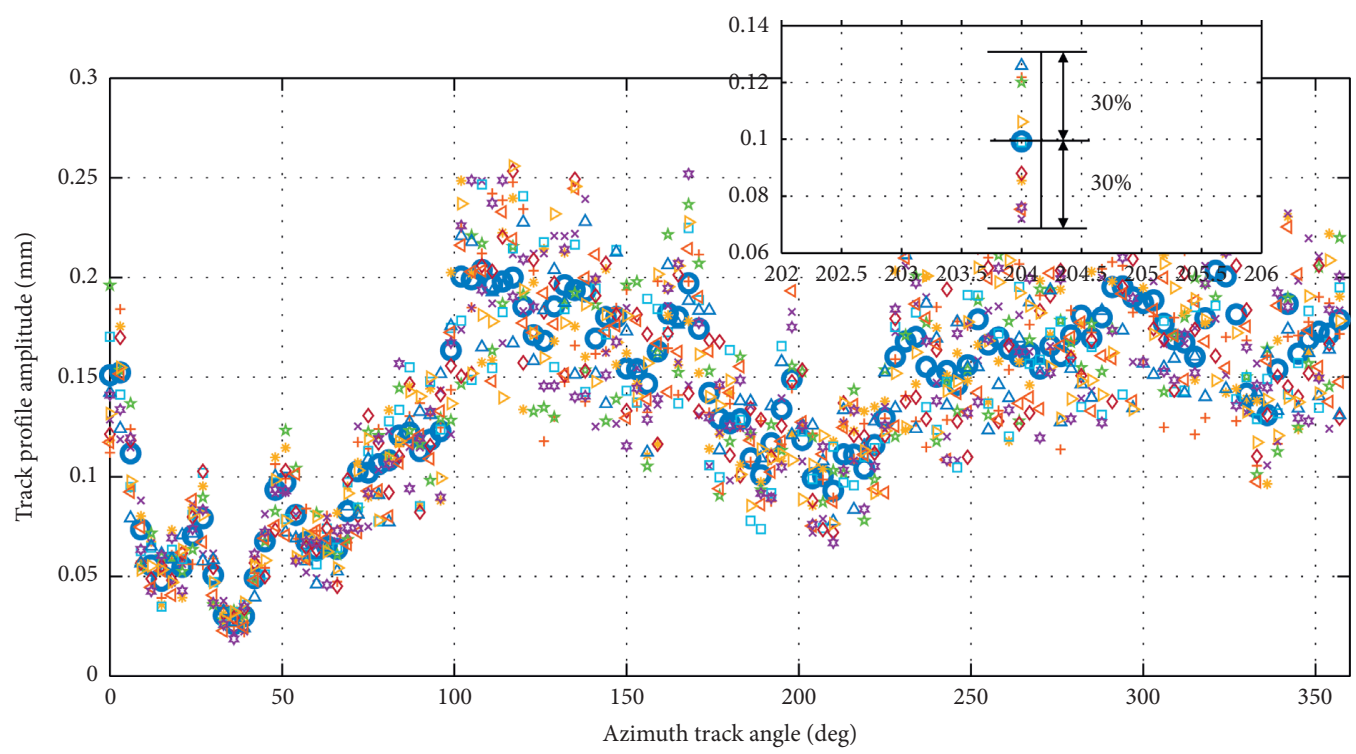

Figure 16: The assumed azimuth track profile distribution with a $30 \%$ deviation compared to the original raw data.

TABLE 1: The summary of the RMS value for different support numbers.

\begin{tabular}{lccc}
\hline No. & 4 support & 6 supports & 8 supports \\
\hline 1 & 0.0058 & 0.0043 & 0.0072 \\
2 & 0.0062 & 0.0045 & 0.0047 \\
3 & 0.0063 & 0.0049 & 0.0061 \\
4 & 0.0061 & 0.0041 & 0.0070 \\
5 & 0.0056 & 0.0042 & 0.0060 \\
6 & 0.0061 & 0.0041 & 0.0054 \\
7 & 0.0054 & 0.0045 & 0.0053 \\
8 & 0.0053 & 0.0045 & 0.0056 \\
9 & 0.0057 & 0.0040 & 0.0056 \\
10 & 0.0062 & 0.0046 & 0.0070 \\
\hline
\end{tabular}

In the modelling process, the whole telescope frame is assumed to be rigid, and the deformation caused by the local contact between the telescope's wheel and the track is not considered in the manuscript. Of course, the consideration of this effect can potentially change the pointing error signature for different support cases greatly, and the conclusion in this research may be altered. The additional consideration of the local deformation can be added to the measured track profile raw data, and together, they will form a new $\mathbf{N}_{\mathrm{A}}^{\mathrm{i}}$ profile matrix in (26), and then the pointing error caused by the track profile and the local deformation can be calculated correspondingly. However, the accurate evaluation of this local deformation needs the Finite Element Model of the telescope structure or the measurement of the local deformation, which is out of the scope of this research project.

Most telescope designs around the world have adopted the 4-support solution, and telescope structure designers choose this design because the 4-support design is the simplest for the design and the cheapest for the budget. Therefore, as we can see that their decision has been heavily impacted by the economic consideration instead of the performance of the telescope pointing error. As shown in this research, the selection of the support point number can be different for different track profiles. Even though the "six-support-point case" is found to be optimal in this research with the specified track profiles, the "six-support-point case" choice would be reconsidered when the track profile is changed to another scenario. However, the finding also leads to the following suggestions:

(a) When the telescope designer starts a new design to ask how many supports should they adopt, they should talk to the track designer as well as the track manufacturer and ask about the precision for the track design and manufacture. They should give a track profile precision range, based on which the telescope designer can select the telescope support number from the view of the reducing the telescope pointing error.

(b) Once the telescope support number is decided and the telescope is built, for example, 4 or 6 , the maintenance engineering should monitor the track profile change, and once it deviates from its design range, actions should be taken to make sure the profile is within the design range.

Further, we have tested another ten different rail track profiles, whose value is assumed to be a random value within a 30\% range deviation compared with the original raw data, as shown in Figure 16. The predicted pointing errors under these ten rail track profiles for different support numbers have also been investigated for the case when $\mathrm{EL}=1 \mathrm{deg}$. The RMS values for these pointing errors for different support numbers have been summarized in Table 1. As indicated in the table, the RMS value for these ten cases is consistent with the result in this research paper, where the pointing error for 6 supports is the smallest for all the investigated cases. 


\section{Conclusion}

The telescope pointing error caused by the irregular azimuth track is actually an open-loop control of the RF beam position compared with the control of the pointing error. Even though the look-up table method has been adopted widely during the telescope operation to reduce the repeatable part of the pointing error, there is still a need and a challenge to reduce the telescope error further not only during the operation stage but also in the early design stage. This paper has investigated a large radio telescope support point number effect on its pointing accuracy, including the four-support-point case, the six-support-point case, and the eight-support-point case from the telescope early design aspect. A general telescope pointing error estimation formula has been proposed, where the elevation position matrix, the azimuth position matrix, and the azimuth profile matrix can be constructed for different telescope support point cases. The telescope pointing error root mean square value is used in this research to quantify the effect of the telescope support point numbers on the pointing accuracy. It was found that the telescope support point numbers do have a significant impact on its pointing accuracy, and the relationship between these two is seen as a complex trend as the six-support-point design is found to be optimal in terms of reducing the telescope pointing error in this research. When the support point number increases to eight, the telescope pointing error root mean square value is the highest. All the results can provide a useful guideline for reducing the telescope pointing error in the initial design stage.

\section{Data Availability}

The data are available upon request to the corresponding author.

\section{Conflicts of Interest}

All authors declare that there are no conflicts of interest.

\section{Acknowledgments}

This work was supported by the Natural Science Foundation of Shaanxi Province under No. 2020JQ290, the National Natural Science Foundation of China under No. 51805399, Youth Science and Technology Star Project of Shaanxi Province under No. 2018KJXX-047, and Youth Innovation Team of Shaanxi Universities under No. 201926.

\section{References}

[1] H. J. Kärcher, "Experience with the design and construction of huge telescope pedestals," Proceedings of the SPIE 5382, Second Backaskog Workshop on Extremely Large Telescopes, vol. 5382, pp. 233-244, 2004.

[2] W. Gawronski, "Control and pointing challenges of large antennas and telescopes," IEEE Transactions on Control Systems Technology, vol. 15, no. 2, pp. 276-289, 2007.

[3] J. Ruze, "The effect of aperture errors on the antenna radiation pattern," Nuovo Cimento, vol. 9, no. 3, pp. 364-380, 1952.
[4] S. Von Hoerner, "The design and improvement of tiltable radio telescopes," Vistas in Astronomy, vol. 20, no. 3, pp. 411-444, 1977.

[5] B. Y. Duan and C. S. Wang, "Reflector antenna distortion analysis using MEFCM," IEEE Transactions on Antennas and Propagation, vol. 57, no. 10, pp. 3409-3413, 2009.

[6] Y. R. Samii and R. L. Haupt, "Reflector antenna developments: a perspective on the past, present, and future [J]," IEEE Antennas and Propagation Magazine, vol. 57, no. 2, pp. 85-95, 2015.

[7] W. Gawronski, B. Bienkiewicz, and R. E. Hill, "Wind-induced dynamics of a deep space network antenna," Journal of Sound and Vibration, vol. 178, no. 1, pp. 67-77, 1994.

[8] W. Gawronski, "Modeling wind-gust disturbances for the analysis of antenna pointing accuracy," IEEE Antennas and Propagation Magazine, vol. 46, no. 1, pp. 50-58, 2004.

[9] J. W. M. Baars, B. G. Hooghoudt, A. Greve, and J. Penalver, "Thermal control of the IRAM 30-m millimeter radio telescope," Astronomy and Astrophysics, vol. 195, pp. 364-371, 1988.

[10] A. M. Bayley, R. J. Davis, J. S. Haggis, and H. Karcher, "Thermal effects on the pointing of the 32-m MERLIN radio telescope at Cambridge," Astronomy and Astrophysics, vol. 283, p. 1051, 1994.

[11] J. Antebi and F. W. Kan, "Precision continuous high-strength azimuth track for large telescopes," Proceedings of the SPIE: Future Giant Telescopes, vol. 4840, pp. 612-623, 2003.

[12] W. Gawronski, F. Baher, and O. Quintero, "Azimuth-track level compensation to reduce blind-pointing errors of the deep space network antennas," IEEE Antennas and Propagation Magazine, vol. 42, no. 2, pp. 28-38, 2000.

[13] Y. J. Li, Y. S. F. Aili, and Z. L. Zhang, "Antenna track deformation precise measurement and pointing error model," Geomatics and Information Science of Wuhan University, vol. 38, no. 2, pp. 176-180, 2013.

[14] S. G. Wang, The Analysis and Experiment on Wheel Rail Contact of Radio Telescope, Master thesis, Hangzhou Dianzi University, Hangzhou, China, 2014.

[15] D. R. Smith, "Achievable alignment accuracy and surface hardness of a large welded azimuth track," Proceedings of SPIE: Optomechanical Technologies for Astronomy, vol. 6273, Article ID 627314, 2006.

[16] N. Li, J. Wu, B.-Y. Duan, and C.-S. Wang, "Effect of the rail unevenness on the pointing accuracy of large radio telescope," Acta Astronautica, vol. 132, pp. 13-18, 2017.

[17] W. Gawronski, F. Baher, and E. Gama, "Track level compensation look-up table improves antenna pointing precision," Proceedings of SPIE: Optomechanical Technologies for Astronomy, vol. 6273, Article ID 627316, 2006.

[18] L. Bartosz, "Improving pointing of Torun 32-m radio telescope: effects of rail surface irregularities," Experimental Astronomy, vol. 45, pp. 81-105, 2018.

[19] D.-Q. Kong, S.-G. Wang, J.-Q. Wang, M. Wang, and H.-B. Zhang, "A new calibration model for pointing a radio telescope that considers nonlinear errors in the azimuth axis," Research in Astronomy and Astrophysics, vol. 14, no. 6, pp. 733-740, 2014.

[20] Z. P. Chen, D. Q. Kong, C. G. Li, N. J. Zhu, and H. L. Shi, "Mechanical analysis and measurement of wheel-rail contact system in large aperture radio telescope," Proceedings of SPIE: Ground-Based and Airborne Telescopes, vol. 9906, Article ID 99064B, 2016.

[21] A. Symmes, R. Anderson, and D. Egan, "Improving the service life of the 100-meter green bank telescope azimuth track," 
Proceedings of SPIE: Ground-Based and Airborne Telescopes II, vol. 7012, pp. 37-49, 2008.

[22] J. Baars and J. K. Hans, Radio Telescope Reflectors: History Development of Design and Construction, Springer, Berlin, Germany, 2018.

[23] W. K. Frank and A. Joseph, "Slip and wear in multilayer azimuth track systems," Proceedings of SPIE: Astronomical Structures and Mechanisms Technology, vol. 5495, pp. 549587, 2004.

[24] W. Gawronski, Modelling and Control of Antennas and Telescopes, Springer, Berlin, Germany, 2008.

[25] J. J. Condon, GBT Pointing Equations, GBT Tech Solutions Private Limited, Kolkata, India, 1992. 